\title{
Dynamical bunching and density peaks in expanding Coulomb clouds
}

\author{
B. S. Zerbe, ${ }^{*}$ X. Xiang, C.-Y. Ruan, S. M. Lund, and P. M. Duxbury ${ }^{\dagger}$ \\ Department of Physics and Astronomy, Michigan State University, \\ 220 Trowbridge Road, East Lansing, Michigan 48824, USA
}

(Received 22 December 2017; published 14 June 2018)

\begin{abstract}
Expansion dynamics of single-species, non-neutral clouds, such as electron bunches used in ultrafast electron microscopy, show novel behavior due to high acceleration of particles in the cloud interior. This often leads to electron bunching and dynamical formation of a density shock in the outer regions of the bunch. We develop analytic fluid models to capture these effects, and the analytic predictions are validated by PIC and N-particle simulations. In the space-charge dominated regime, two and three dimensional systems with Gaussian initial densities show bunching and a strong shock response, while one dimensional systems do not; moreover these effects can be tuned using the initial particle density profile and velocity chirp.
\end{abstract}

DOI: 10.1103/PhysRevAccelBeams.21.064201

\section{INTRODUCTION}

Non-neutral plasma systems arise in a variety of physical contexts ranging from astrophysics [1-3]; accelerator technologies [4-7]; ion and neutron production [8-13]; sources for electron and ion microscopy [14,15]; to high power vacuum electronics [16-18]. Understanding of the dynamics of spreading of such systems is critical to the design of next generation technologies, and simple analytic models are particularly helpful for instrument design. As a result, substantial theoretical efforts have already been made in this vein [19-30]. Specifically, free expansion of clouds of charged single-specie particles starting from rest have been well studied both analytically and computationally [15,21,23,26,27,31-39], and a number of studies have found evidence of the formation of a region of highdensity, often termed a "shock," on the periphery of the clouds under certain conditions [14,23-26,31,34].

One application of these theories that is of particular current interest is to high-density electron clouds used in next-generation ultrafast electron microscopy (UEM) development [40-42]. The researchers in the UEM and the ultrafast electron diffraction (UED) communities have conducted substantial theoretical treatment of initially extremely short bunches of thousands to ultimately hundreds of millions of electrons that operate in a regime dominated by a virtual cathode (VC) limit [36,40,43-45] which is akin to the Child-Langmuir current limit for beams

\footnotetext{
*zerbe@msu.edu

duxbury@msu.edu
}

Published by the American Physical Society under the terms of the Creative Commons Attribution 4.0 International license. Further distribution of this work must maintain attribution to the author(s) and the published article's title, journal citation, and DOI. generated under steady-state conditions [18]. These short bunches are often generated by photoemission, and such bunches inherit an initial profile similar to that of the driving laser pulse profile. Typically, the laser pulse has an in-plane, "transverse" extent that is of order one hundred microns and a duration on the order of fifty femtoseconds, and these parameters translate into an initial electron bunch with similar transverse extents and sub-micron widths [40]. After photoemission, the electrons are extracted longitudinally using either a DC or AC field typically in the 1-10 MV/m [46-49] through tens of $\mathrm{MV} / \mathrm{m}$ [50-52] ranges, respectively. However, the theoretical treatments of such "pancake-like" electron bunch evolution have largely focused on the longitudinal dimension [32-35, 44]. The few studies looking at transverse dynamics have either assumed a uniform-transverse distribution [35] or have looked at the effect of a smooth Gaussian-to-uniform evolution of the transverse profile on the evolution of the pulse in the longitudinal direction [34,37]. Of specific note, only one analytic study found any indication, a weak longitudinal signal, of a shock [34].

On the other hand, an attractive theoretical observation is that an ellipsoidal cloud of cool, uniformly distributed charged particles has a linear electric field within the ellipsoid which results in maintenance of the uniform charge density as the cloud spreads [23]. In the accelerator community, such a uniform distribution is a prerequisite in employing techniques such as emittance compensation [53] as well as forming the basis of other theoretical analyses. It has long been proposed that such a uniform ellipsoid may be generated through proper control of the transverse profile of a short charged-particle bunch emitted from a source into vacuum [44], and experimental results have shown that an electron cloud emitted from a photocathode and rapidly accelerated into the highly-relativistic regime can develop into a final ellipsoidal profile characteristic of a 


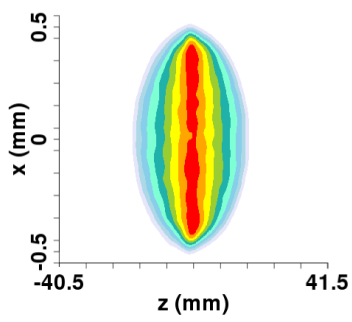

(a) full $z-x$

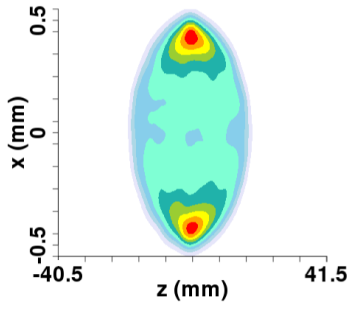

(c) sliced $\mathrm{z}-\mathrm{x}$

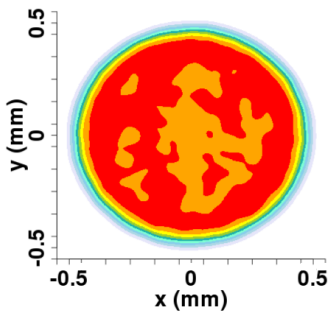

(b) full $\mathrm{x}-\mathrm{y}$

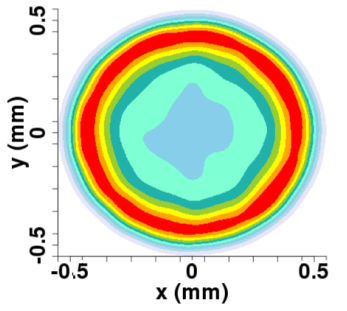

(d) sliced $\mathrm{x}-\mathrm{y}$

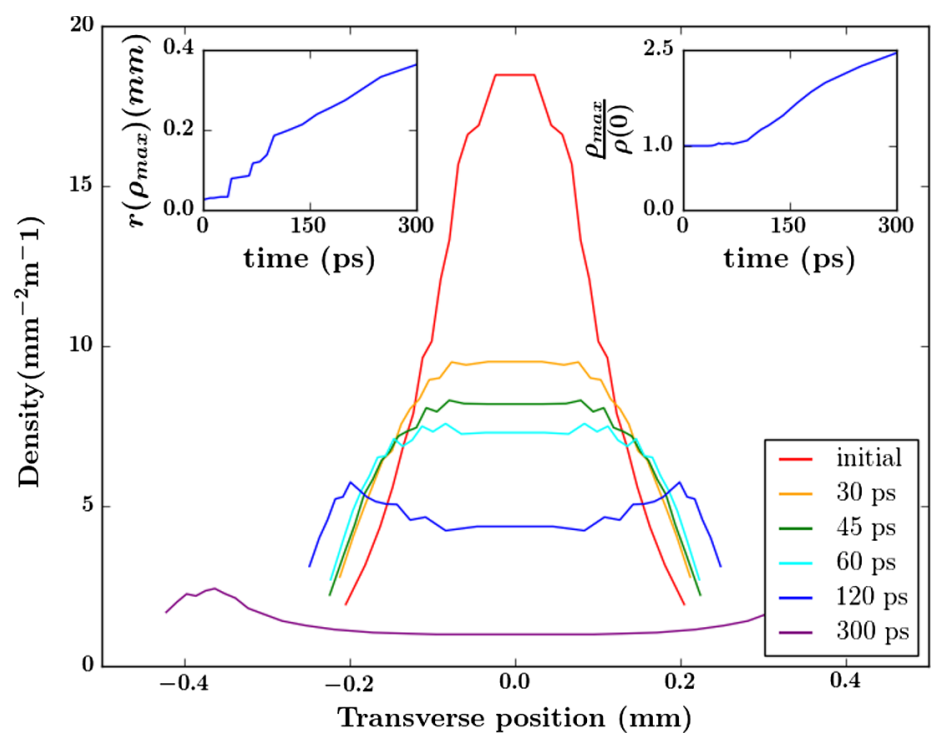

(e) sliced radial evolution

FIG. 1. (a-d) Two dimensional projections of $1 \times 10^{6}$ electron positions simulated with the electrostatic N-particle code, COSY, for approximately $300 \mathrm{ps}$ after injection with a Gaussian $\left(\sigma_{r}=100 \mu \mathrm{m}\right)$ transverse profile into a cavity with an electric field of $10 \mathrm{MV} / \mathrm{m}$. Colors from white to red indicate electron density on a linear scale. (a) and (b) are projections of the full distribution to the $\mathrm{x}-\mathrm{z}$ and $\mathrm{x}-\mathrm{y}$ planes, respectively. (c) and (d) are x-z and x-y projections, respectively, of just the portion of the distribution within $20 \%$ of the standard deviation of the median value of $y$ and $z$, respectively. Notice the ring-like substructure that is evident in the "slices," (c) and (d), but absent from the full distribution projections, (a) and (b). (e) N-particle radial-distributions obtained near the longitudinal median plane of the bunch at various times. Density is calculated by binning 1000 macroparticles and assigning the resulting density to the average radial position of those particles. The initial distribution is sampled from a Gaussian, and the square-like nature of the plot results from the discreteness of the bins. The sub-graph in the upper left corner shows the position of maximum density as a function of time, which is non-zero at initial time due to binning resulting in a non-zero minimum radial position. The subgraph in the upper right shows the ratio of the maximum density to the density at the minimum $r$ value. Notice, the "phase transition" in the $45-80$ ps range where the location of the nonzero, nonstochastic peak first appears well away from the origin.

uniform charge distribution [54]. Contrary to expectations from the free expansion work but consistent with the longitudinal analyses, this profile lacks any indication of a peripheral region of high-density shocks. However, recent work has indicated that a substantial high density region may indeed form in the transverse direction [55], and $\mathrm{N}-$ particle simulation results, as demonstrated in Fig. 1, demonstrate a rapidly developed substantial ringlike shock circumscribing the median of the bunch when a bunch with initial Gaussian transverse-profile starts from sufficient density. Moreover, this shock corresponds to a region of exceedingly low brightness, or conversely, high, local temperature, and experiments show that removal of this region results in a dramatic increase in the bunch brightness [55]. We term this effect "Coulomb cooling" since it is similar to evaporative cooling as the "hottest" charged particles are removed from the distribution's edge thus leaving behind a higher-quality, cooler bunch.

To begin to understand Coulomb cooling, we first investigate this transverse shock. Here we demonstrate the formation of a ring-like shock within N-particle simulations $[56,57]$ of electron bunches with initial transverse Gaussian profile and offer an explanation of why this phenomena has not been noted previously within the UED literature. We then utilize a Poisson fluid approach to derive analytic predictions for the expansion dynamics in planar (1D), cylindrical, and spherical geometries, and we derive conditions for the emergence of density peaks distinct from any initial density maximum. We show that peak formation has a strong dependence on dimension, with one dimensional systems less likely to form shocks, while in cylindrical and spherical geometries bunching is more typical. Particle-incell (PIC) methods, utilizing Warp [58], and N-particle simulations are then used to validate the analytical predictions for peak emergence.

\section{OBSERVATION OF TRANSVERSE SHOCK}

One reason that a transverse shock has not been seen previously in simulations is apparent in Fig. 1 where data was generated using electrostatic N-particle simulations. We consider pancake electron bunches typical of $100 \mathrm{keV}$ ultrafast electron microscopy, and we consider the thin direction of the bunch to be the z-axis. Previous studies of the expansion dynamics of these bunches, including our own work, have looked at the projection of the particle density distribution to the $\mathrm{x}-\mathrm{z}$ plane $[38,44,54,59,60]$. Figure 1 shows that by projecting the distribution in this 


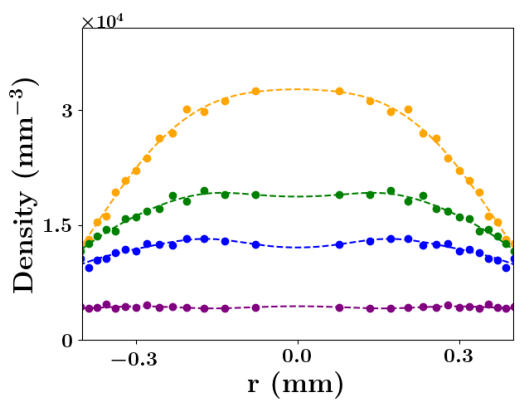

(a) $N=1,000$

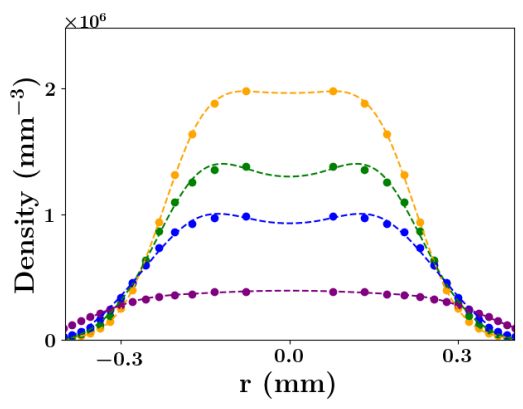

(b) $N=10,000$

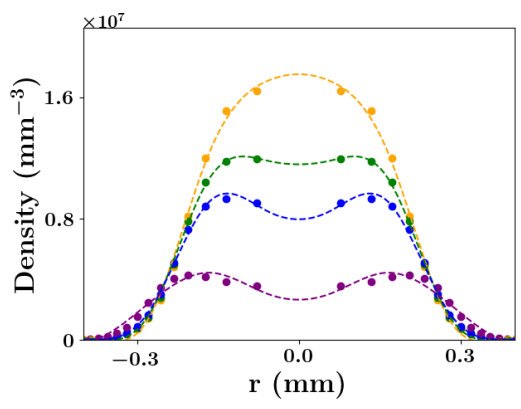

(c) $N=100,000$

FIG. 2. Average density near the z-median of 30 simulations calculated from bunch profiles evaluated at different times: $5 \tau_{p}$ (yellow), $6 \tau_{p}$ (green), $7 \tau_{p}$ (blue), and $10 \tau_{p}$ (purple) where $\tau_{p}=2 \pi \sqrt{\frac{m \epsilon_{0}}{n e^{2}}}, n=\frac{N}{\pi \sigma_{r}^{2} \sigma_{z}}, \sigma_{r} \approx 100 \mu \mathrm{m}$, and $\sigma_{z} \approx 0.4 \mu \mathrm{m}$, for different values of the total number of electrons, $N$. Dotted lines represent spline fits of order 3 with 10 knots.

manner, and with the ability to statistically discern density fluctuations at about the $10 \%$ level, results in what appears to be a uniform distribution; however, by restricting the projection to only electrons near the median of the bunch, a restriction that can only be done computationally presently, results in evidence of a transverse ringlike density substructure near the median longitudinal (z) position.

To better understand when this shock emerges, simulations with the same distribution parameters $\left(\sigma_{r} \approx 100 \mu \mathrm{m}\right.$ and $\Delta z \approx 0.4 \mu \mathrm{m}$ ) but various numbers of electrons were run. The average radial density was calculated for 30 instances of bunches with 1 thousand, 10 thousand, and 100 thousand electrons. As can be seen in Fig. 2, the shock emergence is present for bunches with 100 thousand electrons but not for those with 1 thousand electrons. The case of bunches with 10 thousand electrons suggests the emergence of the shock, but the shock becomes less defined at later times. Figure 3 shows six density profiles at time $10 \tau_{p}$, where $\tau_{p}$ represents the plasma period, with $\tau_{p}=2 \pi \sqrt{\frac{m \epsilon_{0}}{n e^{2}}}$ and $n=\frac{N}{\pi \sigma_{r}^{2} \sigma_{z}}$. The dots in each figure indicate average densities in cylindrical rings, originating from two randomly chosen initial conditions (figures in each row) and for three values of the total number of electrons $N$ : 1 thousand, 10 thousand, and 100 thousand electrons; for the top, middle, and bottom rows, respectively. These representative density plots support the conclusion that the shock is only present in the case of bunches with 100 thousand electrons; where the spline fit to the data indicates a significant peak removed from the center of the bunch in all instances examined. As expected, the density of bunches with one thousand electrons is noisy due to low statistics both from the small number of electrons in the simulation and the large proportion of electrons that spread beyond the analysis region due to the initial velocity spread. Further, the density profile of bunches with 10 thousand electrons has a consistent general shape that conforms well to the spline fit but lacks significant emergent peaks, the indicators of shock formation.

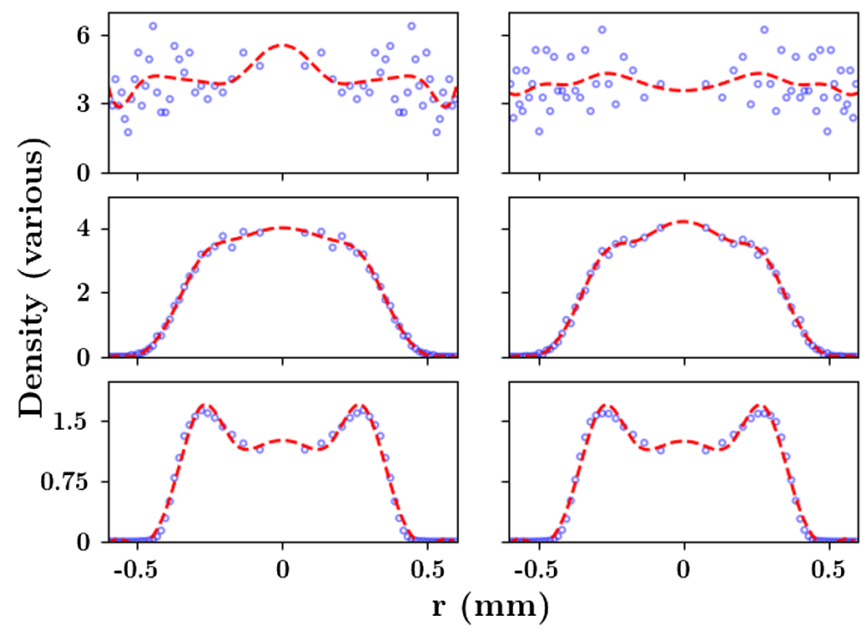

FIG. 3. Density near the z-median of simulated pancake bunches with transverse Gaussian profiles $\left(\sigma_{r}=100 \mu \mathrm{m}\right)$ in an extraction field of $10 \mathrm{MV} / \mathrm{m}$. Each row is the transverse radial density of a section of width $\sigma_{z} \approx 0.4 \mu \mathrm{m}$ for different numbers of electrons, and columns represent 2 different initial conditions. Density was calculated at time $10 \tau_{p}$ where $\tau_{p}=$ $2 \pi \sqrt{\frac{m \epsilon_{0}}{n e^{2}}}$ is the plasma frequency and where $n=\frac{N}{\pi \sigma_{r}^{2} \sigma_{z}}$. The number of electrons in each horizontal panel is different and equal to $N=1000$ (top), $N=10000$ (middle), and $N=100000$ (bottom). For the density at $10 \tau_{p}, 30$ cylindrical shells of equal volume and length $\sigma_{z}$ partitioned the distribution out to $0.6 \mathrm{~mm}$, and the numbers of electrons in each of these shells were used to calculate a density at the shell's average radius. Due partially to the different numbers of electrons and partially due to the fact that the longer simulations, namely the simulation with $N=1000$, resulted in significantly more electrons migrating out of the analysis region as a result of the initial velocity spread, the density scales are different for the three rows in the figure: $\frac{1}{(0.1 \mathrm{~mm})^{3}}$ for the top row, $\frac{0.1}{(0.01 \mathrm{~mm})^{3}}$ for the middle row, and $\frac{1}{(0.01 \mathrm{~mm})^{3}}$ for the bottom row. Red dashed lines represent splines of order 3 with 10 knots. Notice the clear presence of a shock for the case $N=100000$, an ambiguous shock at $N=10000$, and essentially noise at $N=1000$. 


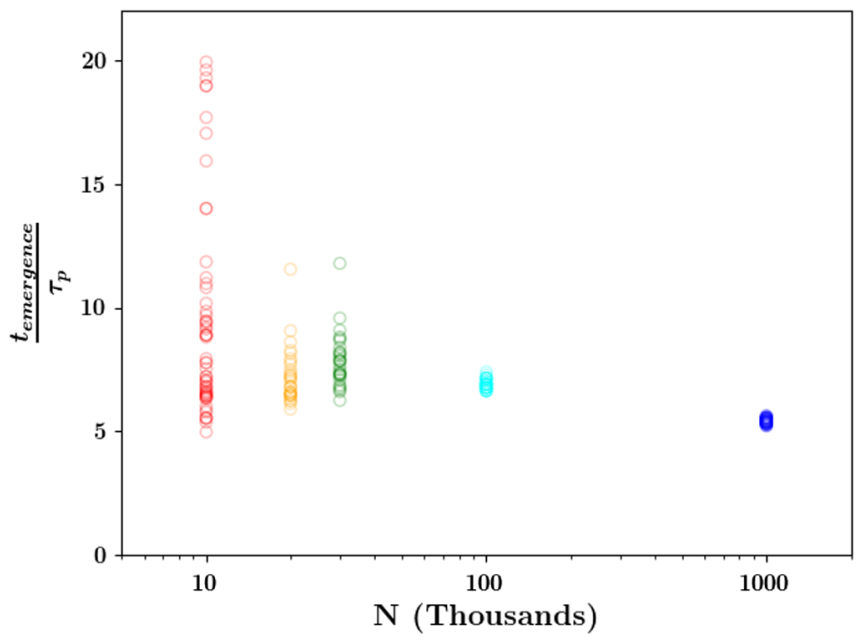

FIG. 4. The emergence time divided by the plasma period as a function of the number of electrons in the initial Gaussian profile with $\sigma_{r} \approx 100 \mu \mathrm{m}$ and $\sigma_{z} \approx 0.4 \mu \mathrm{m}$. Emergence time was determined as the first time the density away from the inner-mostvalue exceeded the inner-most-value by $2 \%$. Notice that the emergence time converges to about $5 \tau_{p}$ for high densities, but at low densities the emergence time has high variability with a median shifted to higher multiples of the plasma period.

We define the emergence time as the time at which peaks indicative of a shock emerge in the dynamics of Coulomb clouds. Figure 4 shows the dependence of the emergence time, and its variability, on the number of electrons in a bunch. It also shows very clearly that the emergence time is proportional to the plasma period, $\tau_{p}$. As can be seen in this figure, the spread in the emergence time is large for a bunch with 10 thousand electrons, but this spread decreases as the density of the bunch increases. For bunches with $N \geq$ 100000 the spread in the emergence time is small, moreover the emergence time appears to converge toward approximately $5 \tau_{p}$ at large $N$ (for Gaussian initial distributions). We note here that for Gaussian pulses with similar spatial and temporal extents, simulations at and above 10 million electrons, a goal of the community [61], result in relativistic velocities as a result of the stronger space-charge effects. As the discussion here focuses on non-relativistic physics, we present data for up to 1 million electrons, where density shock formation occurs well before the electron velocities become relativistic.

The results presented in Figs. 2-4 are a second reason that shock formation has not been seen previously in studies of electron bunches. Specifically, most work has been conducted using $\leq 10000$ electrons with a transverse standard deviation of $100 \mu \mathrm{m}$, and in the regime where there is no consistent emergence of a shock. Moreover, the fact that the non-relativistic evolution of the bunch profile has a time scale proportional to the plasma period, a fact that we derive under special geometries later in this manuscript, means that higher density bunches result in faster, more consistent evolution of the transverse profile.
In other words, the emergence time of a shock happens earlier as the density of the bunch is increased. Specifically, a transverse shock emerges at on the order of $50 \mathrm{ps}$ for an initially Gaussian profile $\left(\sigma_{r}=100 \mu \mathrm{m}\right.$ with sub-micron length) with $10^{6}$ electrons, which is the number of electrons that is the current goal for the diffraction community [61]. This implies that for modern bunches, this transverse shock is happening well within the photoemission gun before the onset of the relativistic regime. The goal of $10^{8}$ electrons for the imaging community needs to be further examined as the transverse velocity spread will be relativistic, but we expect to find this effect there as well; furthermore, we expect that it occurs at short times, of order a few picoseconds.

\section{1D MODEL}

As noted in the introduction, formation of a shock in the longitudinal direction of an expanding pancake pulse has not been observed, and the analysis of Reed [34] demonstrates that this is true for cold initial conditions. Here we rederive this result using an elementary method, which enables extension to include the possibility of an initial chirp; and we find chirp conditions at which shock formation in the longitudinal direction can occur.

Consider the nonrelativistic spreading of an electron bunch in a one dimensional model, which is a good early time approximation to the longitudinal spreading of a pancake-shaped electron cloud generated at a photocathode. For the sake of readability, denote the 1D position of a particle from the Lagrangian perspective to be $z=z(t)$ and $z_{0}=z(0)$. Further, let the cumulative distribution function (cdf)-like parameter $F(z ; t)$ represent the proportion of the distribution to the left of $z$ at time $t$, and define $\delta \sigma(z ; t)=F(z ; t)-F(-z ; t)$. Continuing with the probabilistic analogy, define $\rho=\rho(z ; t)=\frac{d F}{d z}$ to be the normalized density with units of inverse length so that

$$
\delta \sigma=\int_{-z}^{z} \rho(\tilde{z} ; t) d \tilde{z}
$$

Note that $\rho$ is the probabilistic density function (pdf) and is not strictly the charge distribution, $\rho_{q}$. However, the two are related through $\rho_{q}=\Sigma_{\text {tot }} \rho$ where $\Sigma_{\text {tot }}$ is the total charge per unit area. Further, note that as $\Sigma_{\text {tot }}$ is simply a constant, the evolution of $\rho_{q}$ is essentially described by the evolution of $\rho$. Specifically, the acceleration of a Lagrangian particle is

$$
a(z ; t)=\frac{q \Sigma_{\mathrm{tot}}}{2 m \epsilon_{0}} \delta \sigma
$$

where $q$ is the charge of the particle (e.g., electron) and $m$ is its mass. The two key observations enabling analytic analysis are (1) that if the flow of electrons is lamellar, so that there is no crossing of particle trajectories, then 
these integrals and the acceleration calculated from them are time independent and hence may be determined from the initial distribution and (2) that mass is conserved, expressed mathematically by

$$
\rho(z)=\frac{\rho_{0}\left(z_{0}\right)}{z^{\prime}}
$$

where ' denotes $\frac{d}{d z_{0}}$. This allows us to derive an analytic form for the density

$$
\begin{gathered}
\rho(z ; t)=\frac{\rho_{0}}{1+v_{0}^{\prime} t+\frac{q \Sigma_{\text {tot }}}{2 m \varepsilon_{0}} \rho_{0} t^{2}} \\
\frac{d}{d z} \rho(z ; t)=\frac{\rho_{0}^{\prime}\left(1+v_{0}^{\prime} t\right)-\rho_{0} v_{0}^{\prime \prime} t}{\left(1+v_{0}^{\prime} t+\frac{q \Sigma_{\text {tot }}}{2 m \epsilon_{0}} \rho_{0} t^{2}\right)^{3}}
\end{gathered}
$$

Details of these derivations may be found in Appendix A. Equation (5) can be used to show that novel peak formation does not occur in the 1D cases without tuning of the initial position-momentum chirp to a sufficiently negative value. A discussion of this expression and the required conditions on the chirp to cause the emergence of a new density peak are presented in Appendix B.

As we show in the next section the one-dimensional results do not apply, even qualitatively, to higher dimensions, as the constant acceleration situation is not valid and particle crossover can occur even with cold initial conditions. In the next section we present fluid models in higher dimensions where novel mechanisms for emergent density shocks are elucidated.

\section{CYLINDRICAL AND SPHERICAL MODELS}

The derivations for the cylindrical and spherical models are similar so we develop the analysis concurrently in this section.

Consider a nonrelativistic evolving cylindricallysymmetric distribution of charged particles again denoted by $\rho_{q}$. Analogous to the cdf in the planar model, we define the proportion of a distribution contained in a cylinder of radius $r$ at time $t$ to be $\lambda(r ; t)$; analogous to the pdf in the planar model, we denote $\rho$ such that

$$
\lambda(r ; t)=\int_{0}^{r} 2 \pi \tilde{r} \rho(\tilde{r} ; t) d \tilde{r}
$$

where $\rho$ in this model has units of inverse area. Again, the charge density is related to $\lambda$ via $\rho_{q}=\Lambda_{\text {tot }} \rho$ where $\Lambda_{\text {tot }}$ is the constant charge per unit length meaning the entire evolution of $\rho_{q}$ can again be described by the evolution of $\rho$.

Similarly, a non-relativistic evolving sphericallysymmetric distribution of charge particles, $\rho_{q}$, has a unitless cdf, $P(r ; t)$, and pdf, $\rho(r ; t)$, such that

$$
P(r ; t)=\int_{0}^{r} 4 \pi \tilde{r}^{2} \rho(\tilde{r} ; t) d \tilde{r}
$$

Here, $\rho$ has units of inverse volume. Additionally, $\rho_{q}=$ $Q_{\text {tot }} \rho$ where $Q_{\text {tot }}$ is the total charge and the evolution of $\rho_{q}$ and $\rho$ are then again just related by a constant.

In systems with cylindrical and spherically symmetries, the mean field equation of motion for a charge at $r \equiv r(t)$ is given respectively by

$$
\begin{aligned}
& \frac{d \vec{p}}{d t}=\frac{q \Lambda_{\mathrm{tot}} \lambda(r ; t)}{2 \pi \epsilon_{0} r} \hat{r} \\
& \frac{d \vec{p}}{d t}=\frac{q Q_{\mathrm{tot}} P(r ; t)}{4 \pi \epsilon_{0} r^{2}} \hat{r}
\end{aligned}
$$

where $\vec{p}$ is the momentum of a Lagrangian particle. Notice, $r$ in Eq. (8) denotes the cylindrical radius while $r$ in Eq. (9) represents the spherical radius. In both cases, before any crossover occurs, the cdf of a Lagrangian particle is constant in time. For simplicity we write $\lambda(r ; t)=$ $\lambda\left(r_{0} ; 0\right) \equiv \lambda_{0}$ and $P(r ; t)=P\left(r_{0} ; 0\right) \equiv P_{0}$ for a particle starting at $r_{0} \equiv r(0)$. In other words, since $\Lambda_{\text {tot }} \lambda_{0}$ and $Q_{\text {tot }} P_{0}$ can be interpreted as the charge contained in the appropriate Gaussian surface, if we track the particle that starts at $r_{0}$, these contained charges should remain constant before crossover occurs. It is convenient to also define the average particle density to be $\bar{\rho}_{0}=\frac{\lambda_{0}}{\pi r_{0}^{2}}$ in the cylindrically symmetric case and $\bar{\rho}_{0}=\frac{3 P_{0}}{4 \pi r_{0}^{3}}$ in the spherically symmetric case. Likewise, define the initial average charge density as $\bar{\rho}_{q, 0}=\Lambda_{\text {tot }} \bar{\rho}_{0}$ and $\bar{\rho}_{q, 0}=Q_{\text {tot }} \bar{\rho}_{0}$ in the cylindrical and spherically cases, respectively. Notice that these average particle densities are a function solely of $r_{0}$, and we will use these parameters shortly. Equations (8) and (9) may now be rewritten as,

$$
\begin{aligned}
\frac{d p_{r}}{d t} & =\frac{q \Lambda_{\mathrm{tot}} \lambda_{0}}{2 \pi \epsilon_{0} r} \\
\frac{d p_{r}}{d t} & =\frac{q Q_{\mathrm{tot}} P_{0}}{4 \pi \epsilon_{0} r^{2}}
\end{aligned}
$$

for the cylindrical and spherical cases respectively. These equations apply for the period of time before particle crossover. Note that unlike the one dimensional case, in two and three dimensional systems the acceleration on a Lagrangian particle is not constant; time dependence can be seen implicitly in Eqs. (10) and (11) through the time dependent position $r=r\left(r_{0} ; t\right)$ term in the denominator.

Since Eqs. (10) and (11) represent the force on the particle in the cylindrical and spherical contexts, respectively, we can integrate over the particle's trajectory to calculate the change in the particle's energy. Integrating from $r_{0}, 0$ to $r, t$ gives for the cylindrical and spherical cases respectively 


$$
\begin{gathered}
E(r ; t)-E\left(r_{0} ; 0\right)=\frac{q \Lambda_{\mathrm{tot}} \lambda_{0}}{2 \pi \epsilon_{0}} \ln \left(\frac{r}{r_{0}}\right) \\
E(r ; t)-E\left(r_{0} ; 0\right)=\frac{q Q_{\mathrm{tot}} P_{0}}{4 \pi \epsilon_{0}}\left(\frac{1}{r_{0}}-\frac{1}{r}\right)
\end{gathered}
$$

where the term on the right side of the equality can be interpreted as the change in the potential energy due to the self-field of the bunch; within the electrostatic approximation. To test whether the electrostatic approximation is accurate, the particle in cell (PIC) simulations presented in the next section were carried out using both the electrostatic (ES) solver and the fully relativistic electrodynamic (EM) solver. For the cases studied here, no significant difference was found between the results found using these procedures.

In the nonrelativistic limit, we can derive implicit position-time relations for the particle by setting the energy difference equal to the nonrelativisitic kinetic energy, $\frac{1}{2} m v^{2}$, and integrating. The details of this derivation have been placed in Appendix $\mathrm{C}$ and the resulting expressions in the cold-case for the cylindrical and spherical systems are

$$
\begin{aligned}
& t=\frac{\bar{\tau}_{p, 0}}{\pi} \frac{r}{r_{0}} F\left[\sqrt{\ln \left(\frac{r}{r_{0}}\right)}\right] \\
& t=\sqrt{\frac{3}{2}} \frac{\bar{\tau}_{p, 0}}{2 \pi}\left[\tanh ^{-1}\left(\sqrt{1-\frac{r_{0}}{r}}\right)+\frac{r}{r_{0}} \sqrt{1-\frac{r_{0}}{r}}\right]
\end{aligned}
$$

where $F(\cdot)$ represents the Dawson function and $\bar{\tau}_{p, 0}$ represents the plasma period determined from the initial conditions, $\bar{\tau}_{p, 0}=2 \pi \sqrt{\frac{m \bar{\rho}_{0}}{q \bar{p}_{0.0}}}$, indicating that the appropriate time scale is the scaled plasma period. This is consistent with the timescale found in Figs. 2 and 4 for the case of pancake bunches used in ultrafast electron diffraction systems. Equation (15) and its derivation is equivalent to previous time-position relations reported in the literature $[26,62]$ although the previous work did not identify the plasma period as the key time-scale of Coulomb spreading processes and cylindrical symmetry was not discussed [Eq. (14)].

The time-position relations detailed in the equations above depend solely on the amount of charge nearer to the origin than the point in question, i.e., $\bar{\rho}_{q, 0}$, and not on the details of the distribution. Notice however, that it is the difference between the time-position relationships of different locations where the details of the distribution become important and may cause neighboring particles to have interesting relative dynamics; leading to the possibility of shock formation in the density.

To translate the Lagrangian particle evolution equations above to an understanding of the dynamics of the charge density distribution, we generalize Eq. (3) to

$$
\rho(r ; t)=\left(\frac{r_{0}}{r}\right)^{d-1} \frac{\rho_{0}}{r^{\prime}}
$$

where $d$ is the dimensionality of the problem, i.e., 1 (planar symmetry), 2 (cylindrical symmetry), or 3 (spherical symmetry), and ' $\equiv \frac{d}{d r_{0}}$. The factor in the denominator, $r^{\prime}$, may be determined implicitly from the time-position relations above, and the details are presented in Appendix D. The resulting expressions for the density dynamics, in the cold case, for $d=2$ (cylindrical) and $d=3$ (spherical) cases are

$$
r^{\prime}=\frac{r}{r_{0}}\left[1+D_{d}\left(r_{0}\right) f_{\mathrm{d}}\left(\frac{r}{r_{0}}\right)\right]
$$

where

$$
D_{d}=D_{d}\left(r_{0}\right)=\frac{d}{2}\left(\frac{\rho_{0}}{\bar{\rho}_{0}}-1\right),
$$

is a function only of the initial position. The function $f_{2}$ for cylindrical systems is given by

$$
f_{2}\left(\frac{r}{r_{0}}\right)=2 \sqrt{\ln \left(\frac{r}{r_{0}}\right)} F\left[\sqrt{\ln \left(\frac{r}{r_{0}}\right)}\right],
$$

while $f_{3}$ for systems with spherical symmetry is,

$$
f_{3}\left(\frac{r}{r_{0}}\right)=\frac{r_{0}}{r} \sqrt{1-\frac{r_{0}}{r}} \tanh ^{-1}\left(\sqrt{1-\frac{r_{0}}{r}}\right)+1-\frac{r_{0}}{r} .
$$

Note that these are functions of the ratio $r / r_{0}$. The functions $f_{d}$ can also be written as mixed functions of $r$ and $t$, specifically $f_{2}\left(\frac{r}{r_{0}} ; t\right)=\frac{r_{0}}{r} \sqrt{\ln \left(\frac{r}{r_{0}}\right)} \bar{\omega}_{p, 0} t$ and $f_{3}\left(\frac{r}{r_{0}} ; t\right)=\frac{r_{0}}{r} \sqrt{1-\frac{r_{0}}{r}} \bar{\omega}_{p, 0} t \quad$ where $\quad \bar{\omega}_{p, 0}=\frac{2 \pi}{\bar{\tau}_{p, 0}}=\sqrt{\frac{q \bar{p}_{q, 0}}{m \epsilon_{0}}}$. However, care must be used when using these mixed forms as $r$ is implicitly dependent on $t$. Here we work with these functions in terms of relative position, $\frac{r}{r_{0}}$. Substituting Eq. (17) into Eq. (16), we find that the density evolution in systems with cylindrical $(d=2)$ and spherical symmetry $(d=3)$ can be compactly written as

$$
\rho(r ; t)=\left(\frac{r_{0}}{r}\right)^{d} \frac{\rho_{0}}{1+D_{d}\left(r_{0}\right) f_{\mathrm{d}}\left(\frac{r}{r_{0}}\right)}
$$

This expression is general and can be applied to arbitrary, cylindrically, or spherically symmetric initial conditions.

Analogously to the $1 \mathrm{D}$ case the condition, $r^{\prime}<0$ results in particle crossover. However, as detailed in Eq. (17), the sign of $r^{\prime}$ depends on the sign of $1+D_{d}\left(r_{0}\right) f_{\mathrm{d}}\left(\frac{r}{r_{0}}\right)$. It is very interesting to note that $D_{\mathrm{d}}\left(r_{0}\right)$ is the deviation from a uniform distribution function, so that the $D$ functions are 
solely functions of the initial conditions and are positive at locations where the local density is larger than the average density at $r_{0}$ and negative when the local density is smaller than the average density at $r_{0}$. On the other hand, the functions $f_{d}$ are functions of the evolution of the Langrangian particle. One immediate consequence of Eq. (21) is that for a uniform initial density distribution, for either cylindrical or spherical systems, the corresponding $D$ function is zero at every location where the original density is defined. Thus, the uniform density evolution in Eq. (16) reduces to the generally recognized expressions: $\rho(r ; t) \pi r^{2}=\rho_{0} \pi r_{0}^{2}$ for the cylindrical case; and $\rho(r ; t) \frac{4}{3} \pi r^{3}=\rho_{0} \frac{4}{3} \pi r_{0}^{3}$ in the spherical case. We provide additional details for the uniform distribution in the next section. However, Eq. (17) is general for any distribution before particle crossover, not just the uniform distribution.

For a particle starting at position $r_{0}$ and having a deviation from uniform function $D_{\mathrm{d}}\left(r_{0}\right)$, crossover occurs when the particle is at a position, $r$, that satisfies $f_{\mathrm{d}}\left(\frac{r}{r_{0}}\right)=-1 / D_{d}\left(r_{0}\right)$. Since every particle moves toward positive $r$, every particle will have a time for which it will assume every value of the function $f_{d}\left(\frac{r}{r_{0}}\right)$. The characters of $f_{2}$ and $f_{3}$ are similar as can be seen in Fig. (5) where the value of the function is plotted against $\frac{r}{r_{0}}$. Specifically, both functions increase to a maximum and then asymptote towards 1 from above. This means that all density positions eventually experience uniform-like scaling since $\lim _{r \rightarrow \infty} f_{d}\left(\frac{r}{r_{0}}\right)=1$ results in Eq. (16) simplifying to $\rho \pi r^{2}=$ $\rho_{0} \frac{\pi r_{0}^{2}}{1+D_{\mathrm{d}}\left(r_{0}\right)}$ and $\rho \frac{4}{3} \pi r^{3}=\rho_{0} \frac{\frac{4}{3} \pi r_{0}^{3}}{1+D_{\mathrm{d}}\left(r_{0}\right)}$ in the cylindrical and spherical cases, respectively, for large enough $r$. Notice, this uniform-like scaling does not mean that the distribution goes to the uniform distribution, which is what happens in $1 \mathrm{D}$ but need not happen under cylindrical and spherical geometries.

The main difference between the cylindrical and spherical symmetries is that the cylindrical function's maximum is larger than the spherical function's maximum; and we find $\max \left(f_{2}\right) \approx 1.28$ while $\max \left(f_{3}\right) \approx 1.07$. Moreover the maximum of the cylindrical function occurs at a larger value of $\frac{r_{0}}{r}$ than that of the spherical function; specifically $r \approx 9.54 r_{0}$ instead of $r \approx 8.27 r_{0}$, respectively. The first observation means cylindrical symmetry is more sensitive to the distribution than the spherical case, while the second observation indicates that if crossover is going to occur for a specific particle, it will occur before the $r$ value for which the corresponding $f$ function is maximum (i.e., $r \approx 9.54 r_{0}$ or $r \approx 8.27 r_{0}$ ), otherwise the particle will never experience crossover while the model is valid. From this reasoning, we obtain the earliest time for crossover by minimizing the time taken for a trajectory to reach the maximum of the function $f_{d}$, with the crossover constraint $r^{\prime}=0$. This may be achieved by using Lagrange multipliers or by running calculations for a series of values of $r / r_{0}$ to find the

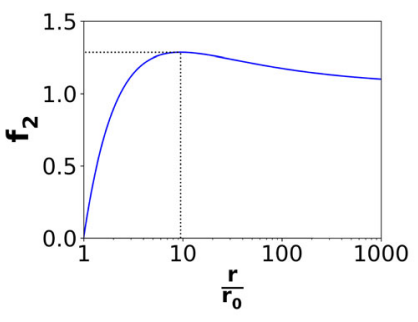

(a) $f_{2}\left(\frac{r(t)}{r_{0}}\right)$

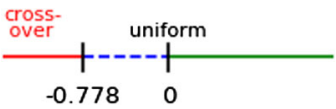

(c) $D_{2}\left(r_{0}\right)$ classification

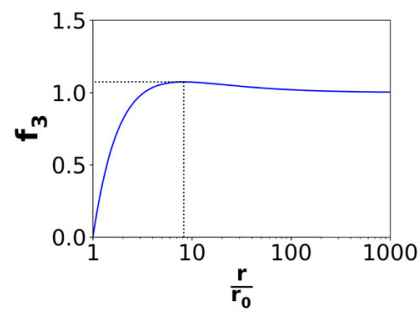

(b) $f_{3}\left(\frac{r(t)}{r_{0}}\right)$

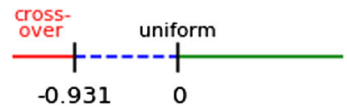

(d) $D_{3}\left(r_{0}\right)$ classification
FIG. 5. (a-b.) Plot of the functions $f_{2}$ and $f_{3}$ against $\frac{r}{r_{0}}$. Since particles are always moving away from the origin, $\frac{r}{r_{0}}>1$ for positive times and this ratio goes to infinity as time goes to infinity. Both functions have a similar character with a maximum in the $8-10 r_{0}$ range, and they both eventually approach one from above. Dashed lines in the plots indicate the location of the functions' maxima. These functions allow us to analytically classify the distribution by the initial value of $D_{\mathrm{d}}\left(r_{0}\right)=$ $\frac{d}{2}\left(\frac{\rho_{0}\left(r_{0}\right)}{\bar{\rho}_{0}\left(r_{0}\right)}-1\right)$ as is done in (c-d). Specifically, crossover may occur at some time when $\frac{d r}{d r_{0}}<0$. For negative values of $D_{d}$ crossover first occurs at the maximum of $f_{d}$ and the value of $D_{d}$ at which that occurs is given in the schematics of $(\mathrm{c}, \mathrm{d})$. For values of $D_{d}$ that are more negative than this value, crossover will occur at some time in some parts of the distribution. For the uniform distribution, $D\left(r_{0}\right)=0$ for all points inside the distribution. The dashed blue line indicates expansion less quickly than the uniform distribution, while the green line indicates more rapid expansion than for the uniform distribution.

position at which crossover happens first. The mean field theory is valid before the minimum crossover time, and the results presented below are well before this time.

\section{UNIFORM AND GAUSSIAN EVOLUTIONS: THEORY AND SIMULATION}

In this section, the mean field predictions are compared to nonrelativistic $\mathrm{N}$-particle simulations, which solve force through electrostatic field superposition, and PIC simulations using an ES solver and relativistic particle pusher. First we present the evolution of the initially-at-rest cylindricallyand spherically-symmetric uniform distribution of $1.875 \times$ $10^{4}$ electrons $/ \mathrm{mm}$ and $2 \times 10^{4}$ electrons within radii's of $1 \mathrm{~mm}$ [see Figs. 6(a) and 6(b)]. Note, in this fairly trivial case, crossover should not occur and the analytic results should be valid mean-field-results for all time. Since $\rho_{0}=\bar{\rho}_{0}$ in this case, $D_{d}\left(r_{0}\right)=0$ and Eq. (21) reduces to

$$
\rho(r ; t)=\left(\frac{r_{0}}{r}\right)^{d} \rho_{0}\left(r_{0}\right)
$$




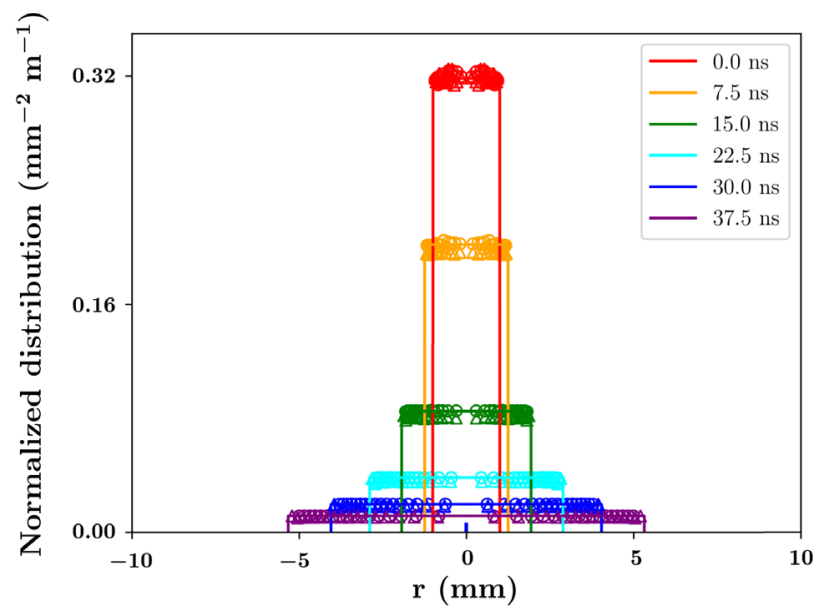

(a) cylindrical symmetry uniform

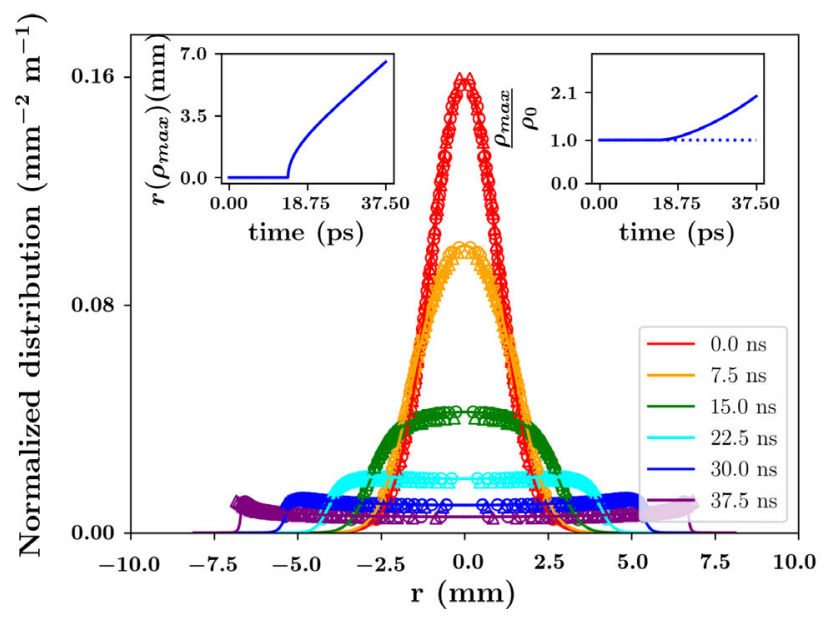

(c) cylindrical symmetry Gaussian

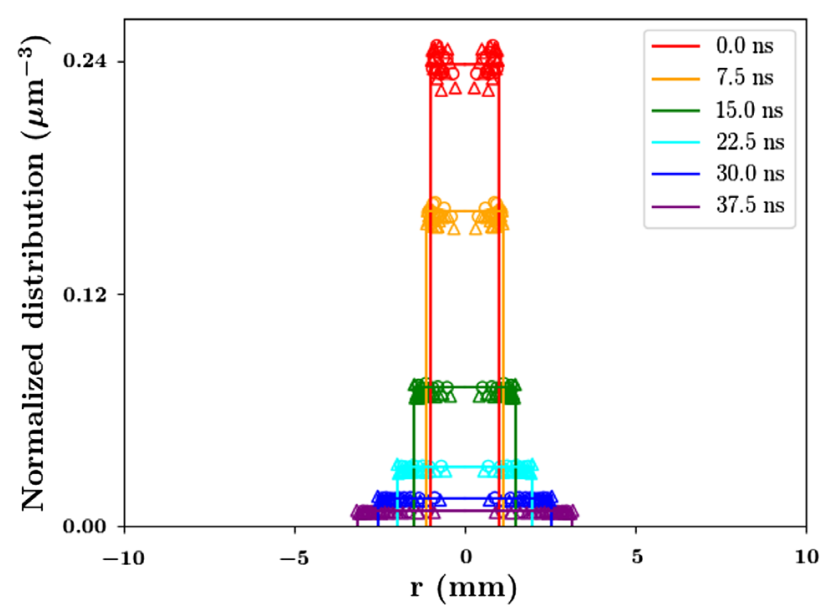

(b) spherical symmetry uniform

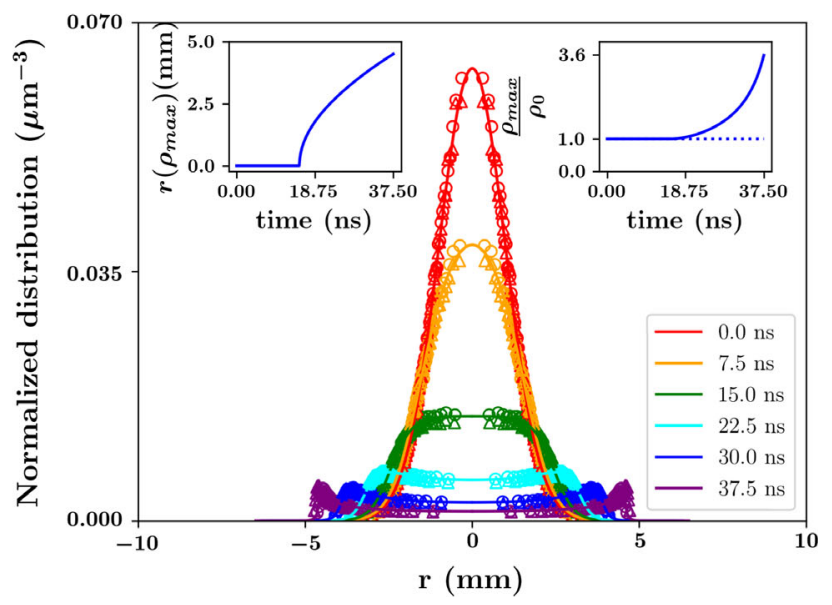

(d) spherical symmetry Gaussian

FIG. 6. Analytical (solid line), PIC (circles) using an ES solver and relativistic particle pusher, and electrostatic N-particle (triangles) results of the normalized density evolution of (a,c) cylindrically and (b, d) spherically symmetric (a, b) uniform and (c,d) Gaussian distributions with $R=\sigma_{r}=1 \mathrm{~mm}$ and $N$ of $1.875 \times 10^{4}$ electrons $/ \mathrm{mm}, 2 \times 10^{4}$ electrons, $3.75 \times 10^{7}$ electrons $/ \mathrm{mm}$, and $10^{5}$ electrons, respectively. The subgraph in the upper left corner of $(\mathrm{c}, \mathrm{d})$ shows the analytic position of max density as a function of time, and the subgraph in the upper right of $(c, d)$ shows the analytic ratio of the max density to the density at the minimum $r$ value both. The corresponding analytic ratio for the uniform distribution is shown in this sub-graph as a dashed horizontal line at 1 . Unsurprisingly, the PIC results and the analytical results, both mean-field models, are in almost perfect agreement, and the N-particle results are in surprisingly good agreement as well. Notice that the models predict peak formation on a time-scale dependent on the initial plasma frequency similar to the peak formation seen in the N-particle disclike density evolution seen in Fig. 1(e) and detailed in Fig. 4.

Notice that $r$ can be solved for a specific time using Eq. (14) or Eq. (15), depending on whether we are examining the cylindrically- or spherically-symmetric case, respectively, and due to $\bar{\rho}_{0}$ 's independence from $r_{0}$, these equations need only to be solved once for a given time to describe all $r$. Therefore, we may write $r=\alpha(t) r_{0} \equiv \alpha r_{0}$, where $\alpha$ is independent of $r_{0}$, and we immediately see that Eq. (22) can be written as $\rho(r ; t)=\alpha^{d} \rho_{0}\left(r_{0}\right)$ suggesting that the density simply scales with time as generally recognized by the community. We solve for $\alpha$ at 6 different times, and present a comparison with both PIC and N-particle cylindrically-symmetric and spherically-symmetric simulations in Figs. 6(a) and 6(b). As can be seen, despite the presence of initial density fluctuations arising from sampling, the simulated results follow the analytic results exceedingly well. Specifically, the distributions simply expand while remaining essentially uniform, and the analytic mean field formulation correctly calculates the rate of this expansion. While this comparison is arguably trivial, it is reassuring to see that our general equation reduces to a form that captures these dynamics.

Less trivial is the evolution of Gaussian distributions. We simulated $3.75 \times 10^{4}$ electrons $/ \mathrm{mm}$ and $10^{5}$ electrons for the cylindrical and spherical cases, respectively, using $\sigma_{r}=1 \mathrm{~mm}$. Solving for the minimum crossover time, we get approximately 44 ns for each distribution. Therefore, we simulate for $37.5 \mathrm{~ns}$, which is well before any crossover events. 
For the Gaussian distributions we introduce the scaled radius variables $s=\frac{r}{\sqrt{2} \sigma_{r}}$ and $s_{0}=\frac{r_{0}}{\sqrt{2} \sigma_{r}}$, so that from Eq. (18) for the cylindrical and spherical cases we have,

$$
\begin{gathered}
D_{2}\left(s_{0}\right)=\frac{\left(1+s_{0}^{2}\right) e^{-s_{0}^{2}}-1}{1-e^{-s_{0}^{2}}} \\
D_{3}\left(s_{0}\right)=\frac{\left(2 s_{0}^{3}+3 s_{0}\right) e^{-s_{0}^{2}}-\frac{3 \sqrt{\pi}}{2} \operatorname{erf}\left(s_{0}\right)}{\sqrt{\pi} \operatorname{erf}\left(s_{0}\right)-2 s_{0} e^{-s_{0}^{2}}}
\end{gathered}
$$

where erf is the well-known error function. Putting these expressions into Eq. (21) we find for the cylindrical and spherical cases respectively

$$
\rho(s ; t)=\frac{\frac{s_{0}^{2}}{\pi s^{2}} e^{-s_{0}^{2}}}{1+2 \frac{\left(1+s_{0}^{2}\right) e^{-s_{0}^{2}}-1}{1-e^{-s_{0}^{2}}} \sqrt{\ln \left(\frac{s}{s_{0}}\right)} F\left[\sqrt{\ln \left(\frac{s}{s_{0}}\right)}\right]}
$$

To find $r(t) / r_{0}$ we solve Eq. (14) or Eq. (15), depending on whether we are examining the cylindrically- or spherically-symmetric cases, respectively; and for $\frac{r}{r_{0}}$, and for every time step, we calculate the predicted distribution at 5000 positions, $r$, corresponding to 5000 initial positions, $r_{0}$, evolved to time $t$. As can be seen in Fig. 6, both the cylindrically- and spherically-symmetric Gaussian distributions develop peaks similar to those seen in the simulations of expanding pancake bunches described in the first section of the paper. As can be seen in Fig. 6, both the PIC and the N-particle results match the analytical results very well. Notice, the primary differences between the cylindrically- and spherically-symmetric evolutions is in their rate of width expansion and the sharpness of the peak that forms, and both of these facets are captured by the analytic models.

\section{DISCUSSION AND CONCLUSIONS}

In this work, we have shown that a shock occurs in the transverse, but not longitudinal, direction during expansion of pancake-like charged particle distributions typical of those used in ultrafast electron microscope (UEM) systems.

Fluid models for arbitrary initial distributions, Eq. (4), a generalization of a model already in the literature, showed that the formation of such a shock should not occur for any cold initial distribution in one dimension. This result is consistent with the finding that typically no shock is visible in the longitudinal direction dynamics of nonrelativistic UEM bunches; however we also showed in Appendix B, that by tuning the initial velocity distribution it is possible to generate a dynamic shock.

We generalized the fluid theory to cylindrical and spherical symmetries deriving implicit evolution equations for the charge density distributions Eq. (21). We analyzed these models for the advent of particle crossover, which occurs for some distributions even when the initial distribution is cold due to the behavior of the Coulomb force in higher dimensions. We found that the time scales associated with the space charge expansion are proportional to the plasma period. One interesting detailed observation is that in the case of cylindrical symmetry, the pre-factor of $\frac{\tau_{p}}{\pi}$ of Eq. (14) is roughly 0.3 while for the spherical symmetric case, corresponding prefactor in Eq. (15), $\sqrt{\frac{3}{2}} \frac{\tau_{p}}{2 \pi}$, is roughly 0.2 plasma periods. Interestingly beam relaxation has been independently found to occur at roughly 0.25 the plasma period [63], which falls directly in the middle of our cylindrically and spherically symmetric models. The analytic theory predicts that emergence of a shock is distribution dependent, and as expected, a uniform initial distribution does not produce a shock. However we showed that electron bunches that are initially Gaussian distributed produce a shock well before the advent of particle crossover indicating that the emergence of a shock is well described by fluid models presented here. This is consistent with the observation of a shock in N-particle simulations of the transverse expansion of UEM pancake bunches (see Figs. 1-4).

To our knowledge, we have presented the first analytic derivation of the cold, single-species, non-neutral density evolution equations for cylindrical and spherical symmetries. These equations are general enough to handle any distribution under these symmetries, and can be used across specialties from accelerator technology, to electronics, to astrophysics. While simulation methods, like the $\mathrm{N}$-particle and PIC codes used here are general tools, the insights provided by these simple analytical equations should provide fast and easy first-approximations for a number of calculations; while providing physical insights and parameter dependences that are more difficult to extract from purely computational studies.

The analysis presented here has been carried out for the nonrelativistic regime; which is only valid for cases of sufficiently low density where the shock occurs prior to the electrons achieving relativistic velocities. For higher densities or other physical situations where the bunch becomes relativistic more quickly than the formation of this shock, a 
relativistic analysis is needed. For example, for sufficiently high densities, e.g., $10^{7}$ or more electrons in a pancake geometry like that used in the typical single shot experiments in the UE field, relativistic effects become important and need to be considered. This will be discussed in detail in a forthcoming paper.

The analysis presented here is based on the force expression, Eqs. (10) and (11), that are derived from Gauss's law. The same physics is contained in the ES solver in Warp and in the N-particle simulation code used here. The excellent agreement between simulations and theory indicates that fluid models describe electron cloud dynamics when electrostatic effects dominate. In the relativistic limit a variety of new effects may become important, including magneotstatic fields, retardation, inertial mass effects and the effects of dynamically varying EM fields. The PIC code Warp supports an electromagnetic (EM) solver that includes all of these effects [64]. This procedure is more computationally expensive but enables a check on the accuracy of the ES approximation; and we used the EM solver in Warp to verify that the results of the ES theory are fully consistent with those of the EM solver in the regimes studied here. Some aspects of the relativisitic limit dynamics have been resolved, specifically, Bychenkov and Kovalev have already included inertial mass effects and ES self-fields in their analysis of the evolution of the uniform distribution [65]. A further question, as noted by an anonymous referee of this paper, is the validity of the standard three step photoemission model which leads to an initial state consisting of initial positions and velocities of a set of $\mathrm{N}$ electrons. In typical simulations, the initial electric field of this set of electrons is calculated electrostatically, and this initial field is used in the simulations, either electrostatically with tools like the ES solver or electrodynamically with tools like the EM solver. Questions should be raised about the need for a dynamical, relativistic treatment of the photoemission process; and this is an important direction for future work especially as the community moves to higher and higher densities that will decrease the time scale of expansion. It is worth noting that a key aspect of a relativistic photoemission model is that it must conserve charge. This has been handled previously by us in the non-relativistic case through incorporating positive "hole" charges on the cathode during electron emission[38], and discussions of the electrodynamics must consider similar processes in order to be physically accurate.

We also point out that the Child-Langmuir current should not have the dynamic shocks elucidated in this paper, except at the onset of the current before the steadystate condition sets in. Previous studies note the "hollowing" of a steady-state beam due to fringe field effects [66], but a steady state Child-Langmuir current is largely independent of emission parameters; so that this hollowing effect is not dynamical, but part of the continuous emission process itself. This is therefore a very different mechanism than the dynamic shocks we see here. It would be interesting to study the combined effects of steady state beam hollowing and dynamic shock formation in pancake bunches to determine if the combination of these processes provides new opportunities for optimization of beam properties.

The analytic models presented here treat free expansion whereas most applications have lattice elements to confine the bunches. Substantial work, in particular the particlecore model, has been very successful at predicting transverse particle halos of beams $[67,68]$. This model assumes a uniform-in-space beam-core density called a KapchinskyVladimirsky (KV) distribution due to its ease of theoretical treatment. Such an assumption is supported by the analysis presented here as we find that the distribution within the shock is nearly uniform. However, the particle-core models do not treat the initial distribution as having a large density on the periphery. It would be interesting to revisit such treatments with this new perspective; specifically, it should be possible to examine the effect of radial-focussing fields on the evolution of the three-dimensional distributions we have investigated here. On the other hand, we would like to point out that the main effect the particle-core model attempts to capture, halos, occur even after aperturing the beam [67] again indicating a different mechanism for the formation of halos than the mechanism of shock formation we describe here.

The experimental work that motivated this analysis, [55], not only predicted a shock but also a correlated decrease in brightness near the periphery. We emphasize that the meanfield equations used here explain the density shock only and do not provide a quantitative theory of the emittance and the Coulomb cooling achieved by removing the electrons in the shock. Specifically, the true emittance in the analytic models presented here remains zero for all time. For instance, in the spherically symmetric case, all particles at a radius $r$ have velocity $\sqrt{\frac{2}{3}} \frac{r_{0}}{\bar{\omega}_{p, 0}} \sqrt{1-\frac{r_{0}}{r}}$ resulting in zero local spread in velocity space. This perfect relationship between velocity and position means that the true emittance is zero even if the relation is nonlinear; however, in such a nonlinear chirp case, the rms emittance will not remain zero despite the true emittance being zero. Moreover, the analytic model does capture some of the rms emittance growth as a change in the distribution has a corresponding change on the variance measures used to determine the rms emittance. Specifically, a Gaussian distribution should have especially large emittance growth due to its evolution to a bimodal distribution, a distribution that is especially problematic for variance measures. Such a large change in the emittance of the transverse Gaussian profile has been seen computationally by Luiten [44] and us $[37,38]$. On the other hand, the perfectly uniform distribution does not change its distribution throughout its evolution and therefore should have zero rms emittance 
growth as the chirp exactly cancels out the expansion of the pulse at all times. Moreover, Luiten et al. found computationally that the uniform distribution does have an increase in emittance although less than the Gaussian case [44], an observation that is corroborated by our own work with PIC and N-particle calculations [37,38]. The analytic formulation of mean-field theory presented here provides new avenues to treating emittance growth, by treating fluctuations to these equations in a systematic manner. This analysis will be presented elsewhere.

\section{ACKNOWLEDGMENTS}

This work was supported by NSF Grant No. 1625181, the College of Natural Science, the College of Communication Arts and Sciences, and the Provost's office at Michigan State University. Computational resources were provided by the High Performance Computer Center at MSU. We thank Martin Berz and Kyoko Makino for their help with employing COSY as an N-particle code. We thank Jean-Luc Vay and Remi Lehe for their help employing the EM solver in Warp. We thank the reviewers of the original version of this paper for their insightful and meticulous comments.

\section{APPENDIX A: 1D DENSITY DERIVATION}

We denote $a(z ; t)=a_{0}, \quad \rho_{0}=\rho\left(z_{0}\right)=\rho(z ; 0)$, and $\delta \sigma=\int_{-z_{0}}^{z_{0}} \rho_{0}(\tilde{z}) d \tilde{z}$. Moreover, due to the fact that for any particle trajectory, the acceleration is constant and given by $a_{0}$, the Lagrangian particle dynamics reduces to the elementary constant acceleration kinematic equation

$$
z(t)=z_{0}+v_{0} t+\frac{1}{2} a_{0} t^{2}
$$

where $v_{0}$ is the initial velocity of the charged particle that has initial position $z_{0}$. Notice that both $v_{0}$ and $a_{0}$ are functions of the initial position, $z_{0}$, and we shall see later that the derivatives of these parameters, $v^{\prime}$ and $a^{\prime}$ where $I \equiv \frac{d}{d z_{0}}$ are important in describing the relative dynamics of Lagrangian particles starting at different initial positions. Moreover, the special case of $v_{0}=0$ everywhere, which we will call the cold-case, is commonly assumed in the literature, and we will examine this case first.

First we consider the spreading of the charge distribution within the Eulerian perspective, where $z$ is an independent variable instead of it describing the trajectory of a particle. We denote the charge distribution at all times to be $\Sigma_{\text {tot }} \rho(z ; t)$ with $\rho(z ; t)$ a unit-less, probability-like density and $\Sigma_{\text {tot }}$ the total charge per unit area in the bunch. Since particle number is conserved, we have

$$
\rho(z ; t) d z=\rho_{0} d z_{0}
$$

so that in the nonrelativistic case derived above,

$$
\rho(z, t)=\frac{\rho_{0}}{z^{\prime}}=\frac{\rho_{0}}{1+v_{0}^{\prime} t+\frac{1}{2} a_{0}^{\prime} t^{2}}
$$

Notice that the derivative of the acceleration with respect to the initial position is directly proportional to the initial distribution, so that,

$$
a_{0}^{\prime}=\frac{q \Sigma_{\mathrm{tot}}}{2 m \epsilon_{0}} \frac{d \delta \sigma}{d z_{0}}=\frac{q \Sigma_{\mathrm{tot}}}{m \epsilon_{0}} \rho_{0}
$$

where we assumed that $\rho_{0}$ is symmetric leading. Plugging Eq. (A4) into Eq. (A3), we get the expression detailed in Eq. (4).

To determine the slope of the density, we take the derivative with respect to the $\mathrm{z}$ coordinate of Eq. (A3)

$$
\begin{aligned}
\frac{d}{d z} \rho(z ; t) & =\left(\frac{\rho_{0}}{z^{\prime}}\right)^{\prime} / z^{\prime} \\
& =\frac{\rho_{0}^{\prime}}{z^{\prime 2}}-\frac{\rho_{0}}{z^{\prime 3}} z^{\prime \prime} \\
& =\frac{\rho_{0}^{\prime} z^{\prime}-\rho_{0} z^{\prime \prime}}{z^{\prime 3}}
\end{aligned}
$$

From the main text, we have

$$
z^{\prime}=1+v_{0}^{\prime} t+\frac{q \Sigma_{\text {tot }}}{2 m \epsilon_{0}} \rho_{0} t^{2}
$$

and from this it is straightforward to show

$$
z^{\prime \prime}=v_{0}^{\prime \prime} t+\frac{q \Sigma_{\text {tot }}}{2 m \epsilon_{0}} \rho_{0}^{\prime} t^{2}
$$

Subbing this back into Eq. (A5), we get

$$
\begin{aligned}
\frac{d \rho}{d z} & =\frac{\rho_{0}^{\prime}\left(1+v_{0}^{\prime} t+\frac{q}{2 m \epsilon_{0}} \rho_{0} t^{2}\right)-\rho_{0}\left(v_{0}^{\prime \prime} t+\frac{q}{2 m \epsilon_{0}} \rho_{0}^{\prime} t^{2}\right)}{\left(1+v_{0}^{\prime} t+\frac{q \Sigma_{\text {tot }}}{2 m \epsilon_{0}} \rho_{0} t^{2}\right)^{3}} \\
& =\frac{\rho_{0}^{\prime}\left(1+v_{0}^{\prime} t\right)-\rho_{0} v_{0}^{\prime \prime} t}{\left(1+v_{0}^{\prime} t+\frac{q \Sigma_{\text {tot }}}{2 m \epsilon_{0}} \rho_{0} t^{2}\right)^{3}}
\end{aligned}
$$

This is Eq. (5) in the main text.

\section{APPENDIX B: 1D CHIRP ANALYSIS}

For the cold-case, Eq. (4) reduces to the density evolution equation derived by Reed [34] using different methods. Also, in the cold-case, Eq. (5) simplifies into a proportionality between the initial slope of the distribution and the slope of the distribution at any later time. Therefore, a charge distribution that is initially at rest and unimodal, i.e., only a single initial location has non-zero density with $\rho_{0}^{\prime}=0$, never develops a dynamically generated second maximum. This explains why we should not expect to see an emergent shock in the longitudinal direction; provided 
the 1D model is applicable and cold initial conditions are valid. However, if a nonlinear velocity chirp exists, i.e., $v_{0}^{\prime \prime} \neq 0$, density peaks will emerge at $z$ when $t=\frac{\rho_{0}^{\prime}}{v_{0}^{\prime \prime} \rho_{0}-v_{0}^{\prime} \rho_{0}^{\prime}}$, and hence occurs at positive time if $v_{0}^{\prime \prime} \rho_{0}>v_{0}^{\prime} \rho_{0}^{\prime}$. In the special case $v_{0}^{\prime \prime} \rho_{0}=v_{0}^{\prime} \rho_{0}^{\prime}$, the distribution may be reframed as a cold-case distribution starting from $t=-\frac{m c_{1} \epsilon_{0}}{q \Sigma_{\text {tot }}}$ for some $z_{0}$-independent constant $c_{1}$ with velocity units when $\frac{m c_{1}^{2} \epsilon_{0}}{q \Sigma_{\text {tot }}}<1$ or a distribution starting from a singularity with velocity distribution $\tilde{v}_{0}=c_{1}\left(\rho_{0}-\frac{1}{2} \delta \sigma\right)$. As noted earlier, the function $a_{0}\left(z_{0}\right)$ is monotonically increasing as a function of distance from the center of the pulse, which means that electrons at the edges of the bunch always have larger accelerations away from the center of the pulse than electrons nearer the pulse center. Thus crossover, where an inner electron moves past an outer electron, cannot occur unless the initial velocities of inner electrons overcome this relative acceleration.

To consider the case where crossover may occur, we add an initial velocity chirp, i.e., $v_{0}=c z_{0}$ where $c$ has units of inverse time. We seek the crossover time, which is the time at which two electrons that were initially apart, are at the same position at a later time. In this case it is straightforward to find the time at which crossover occurs by considering an electron at initial position $z_{0}$, and a second electron at position $z_{0}+\delta z$. Before either of these electrons experiences a crossover Eq. (A1) is valid, and setting $z\left(z_{0}, t_{x}\right)=z\left(z_{0}+\delta z_{0}, t_{x}\right)$ reduces to,

$$
A t_{x}^{2}+B t_{x}+1=0
$$

where $A=\frac{q \Sigma_{\text {tot }}}{2 m \epsilon_{0}} \rho_{0}$ and $B=v_{0}^{\prime}$. Solving the quadratic equation leads to the crossover time given by

$$
t_{x}=\frac{-B \pm \sqrt{B^{2}-4 A}}{2 A}
$$

Since $A$ is always positive, the square root is real only if $B^{2}$ is larger than $4 A$. Moreover the time is only positive if $B$ is negative. Therefore crossover only occurs if the chirp has a negative slope, as expected on physical grounds. The conditions for tuning the chirp to produce crossover in $1 \mathrm{D}$ are then

$$
\begin{gathered}
v_{0}^{\prime}<0 \\
\left|v_{0}^{\prime}\right| \geq \sqrt{\frac{2 q \Sigma_{\mathrm{tot}}}{m \epsilon_{0}} \rho_{0}}
\end{gathered}
$$

The results above are applicable to the spreading in the logitudinal direction of nonrelativistic pancake bunches, because the expression Eq. (A1) is linear in acceleration. In that case, the position of a charged particle at any time can be calculated from a superposition of the contribution from the space-charge field and any external constant field such as a constant and uniform extraction field. In that case, the space charge field leads to spreading of the pulse, while the extraction field leads solely to an acceleration of the center of mass of the entire bunch. In that case the center of mass and spreading dynamics are independent and can be decoupled. The extension of the description above to asymmetric charge density functions is also straightforward, as is the inclusion of an image field at the photocathode. Moreover, inclusion of these effects does not change Eq. (4), Eq. (5), nor the conclusions we have drawn from them. These results apply generally to all times before the initial crossover event within the evolution of the bunch, and once crossover occurs, the distribution can be reset with a new Eq. (4) to follow further density evolution.

\section{APPENDIX C: DERIVATION OF TIME-LOCATION RELATIONS}

\section{Integral form}

Starting with the relativistic expression for change in particle energy derived in the main text

$$
\begin{gathered}
\text { cyl: } E(t)-E(0)=\frac{q \Lambda_{\mathrm{tot}} \lambda_{0}}{2 \pi \epsilon_{0}} \ln \left(\frac{r}{r_{0}}\right) \\
\text { sph: } E(t)-E(0)=\frac{q Q_{\mathrm{tot}} P_{0}}{4 \pi \epsilon_{0}}\left(\frac{1}{r_{0}}-\frac{1}{r}\right)
\end{gathered}
$$

we approximate the energy change with a change in nonrelativistic kinetic energy starting from rest

$$
\begin{gathered}
\text { cyl: } \frac{1}{2} m v^{2}=\frac{q \Lambda_{\mathrm{tot}} \lambda_{0}}{2 \pi \epsilon_{0}} \ln \left(\frac{r}{r_{0}}\right) \\
\text { sph: } \frac{1}{2} m v^{2}=\frac{q Q_{\mathrm{tot}} P_{0}}{4 \pi \epsilon_{0}}\left(\frac{1}{r_{0}}-\frac{1}{r}\right)
\end{gathered}
$$

where $v=\frac{d r}{d t}$ is the velocity of the particle at time $t$ in the two or one of the three dimensional models, respectively, with the appropriate definition of $r$. Solving these equations for the velocity at time $t$, separating the variables and integrating, we obtain

$$
\begin{gathered}
\text { cyl } t=\int_{r_{0}}^{r} \frac{d \tilde{r}}{\sqrt{\frac{q \Lambda_{\text {to }} \lambda_{0}}{\pi m \epsilon_{0}} \ln \left(\frac{\tilde{r}}{r_{0}}\right)}} \\
\operatorname{sph}: t=\int_{r_{0}}^{r} \frac{d \tilde{r}}{\sqrt{\frac{q Q_{\text {to }} P_{0}}{2 \pi m \epsilon_{0} r_{0}}} \sqrt{1-\frac{r_{0}}{\tilde{r}}}}
\end{gathered}
$$

\section{Cylindrically-symmetric integral solution}

We solve the cylindrically-symmetric integral first. First, let $a=\frac{q \Lambda_{\mathrm{tot}}}{\pi m \epsilon_{0}}$. Define $\tilde{u}=\sqrt{a \lambda_{0} \ln \left(\frac{\tilde{r}}{r_{0}}\right)}$. Solving this equation 
for $\tilde{r}$ in terms of $\tilde{u}$, we see that $\tilde{r}=r_{0} e^{\frac{\tilde{u}^{2}}{a \lambda_{0}}}$. It is also straightforward to see that

$$
d \tilde{u}=\frac{1}{\sqrt{a \lambda_{0} \ln \left(\frac{\tilde{r}}{r_{0}}\right)}} \frac{a \lambda_{0}}{2 r_{0}} e^{\frac{-\tilde{u}^{2}}{a \lambda_{0}}} d \tilde{r}
$$

Applying this change of coordinates to Eq. (C5), we get

$$
\text { cyl } t=\frac{2 r_{0}}{\sqrt{a \lambda_{0}}} \int_{0}^{w} e^{\tilde{w}^{2}} d \tilde{w}
$$

where $\tilde{w}=\frac{\tilde{u}}{\sqrt{a \lambda_{0}}}$, and $w=\sqrt{\ln \left(\frac{r}{r_{0}}\right)}$. The remaining integral, $\int_{0}^{w} e^{\tilde{w}^{2}} d \tilde{w}$ can be written in terms of the well-studied Dawson function, $F(\cdot)$ :

$$
\int_{0}^{w} e^{\tilde{w}^{2}} d \tilde{w}=\frac{r}{r_{0}} F\left[\sqrt{\ln \left(\frac{r}{r_{0}}\right)}\right]
$$

Subbing Eq. (C8) back into Eq. (C7) gives us our timeposition relation

$$
\text { cyl: } t=\frac{\bar{\tau}_{p, 0}}{\pi} \frac{r}{r_{0}} F\left[\sqrt{\ln \left(\frac{r}{r_{0}}\right)}\right]
$$

where $\frac{\bar{\tau}_{p, 0}}{2 \pi}=\sqrt{\frac{m \epsilon_{0}}{q \Lambda_{\mathrm{tot}} \bar{\rho}_{0}}}$ and $\bar{\rho}_{0}=\frac{\lambda_{0}}{\pi r_{0}^{2}}$.

\section{Spherically-symmetric integral solution}

We solve the spherically-symmetric integral with an analogous approach. First, let $a=\frac{q Q_{\text {tot }}}{\pi m \epsilon_{0}}$. Define $\tilde{u}=$ $\sqrt{1-\frac{r_{0}}{\tilde{r}}}$ and solving for $\tilde{r}$ gives $\tilde{r}=\frac{r_{0}}{1-\tilde{u}^{2}}$. Thus

$$
d \tilde{u}=\frac{1}{\sqrt{1-\frac{r_{0}}{\tilde{r}}}} \frac{\left(1-\tilde{u}^{2}\right)^{2}}{2 r_{0}} d \tilde{r}
$$

Applying this change of coordinates to Eq. (C6) with $u=\sqrt{1-\frac{r_{0}}{r}}$, we get

$$
\begin{aligned}
& \text { sph: } t=\sqrt{\frac{2 r_{0}}{a P_{0}}} \int_{0}^{u} \frac{2 r_{0}}{\left(1-\tilde{u}^{2}\right)^{2}} d \tilde{u}
\end{aligned}
$$

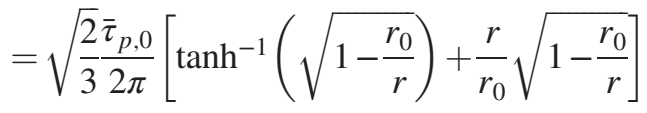

where the solution to the integral was obtained with Mathematica's online tool [69] and where $\bar{\rho}_{0}=\frac{P_{0}}{\frac{4}{3} \pi r_{0}^{3}}$ and $\frac{\bar{\tau}_{p, 0}}{2 \pi}=\sqrt{\frac{m \epsilon_{0}}{q Q_{\mathrm{tot}} \bar{\rho}_{0}}}$.

\section{APPENDIX D: DERIVATION OF DERIVATIVES WITH RESPECT TO INITIAL POSITION}

Denote $I \equiv \frac{d}{d r}$. As noted in the main text, much of the physics of distribution evolution in our models is captured in the term $r^{\prime}$. The procedure to derive the expressions for this derivative is to take the derivative of Eqs. (C9) and (C10), which should be zero, and then solving for $r^{\prime}$. We do this mathematics here.

\section{The cylindrically-symmetric derivative}

First, let $a=\frac{q \Lambda_{\mathrm{tot}}}{\pi m \epsilon_{0}}$. We begin by rewriting $t$ from Eq. (C9) as

$$
t=2 r \sqrt{\frac{1}{a \lambda_{0}}} F(y)
$$

where $y=\sqrt{\ln \left(\frac{r}{r_{0}}\right)}=\sqrt{\ln (r)-\ln \left(r_{0}\right)}$. So

$$
y^{\prime}=\frac{1}{2 y}\left(\frac{r^{\prime}}{r}-\frac{1}{r_{0}}\right)
$$

The Dawson function has the property $\frac{d}{d y} F(y)=$ $1-2 y F(y)=\left(\frac{1}{F(y)}-2 y\right) F(y)$, and with the chain rule this becomes $F^{\prime}(y)=\left(\frac{1}{F(y)}-2 y\right) y^{\prime} F(y)$. Using Eq. (D2), this becomes

$$
F^{\prime}(y)=F(y)\left(\frac{1}{2 y F(y)}-1\right)\left(\frac{r^{\prime}}{r}-\frac{1}{r_{0}}\right)
$$

Also, note

$$
\begin{aligned}
\left(\frac{1}{\sqrt{\lambda_{0}}}\right)^{\prime} & =-\frac{1}{2 \sqrt{\lambda_{0}}} \frac{\lambda_{0}^{\prime}}{\lambda_{0}} \\
& =-\frac{1}{\sqrt{\lambda_{0}}} \frac{1}{r_{0}} \frac{\rho_{0}}{\bar{\rho}_{0}}
\end{aligned}
$$

So

$$
\begin{aligned}
0 & =t^{\prime} \\
& =\frac{t r^{\prime}}{r}-\frac{t}{r_{0}} \frac{\rho_{0}}{\bar{\rho}_{0}}+t\left(\frac{1}{2 y F(y)}-1\right)\left(\frac{r^{\prime}}{r}-\frac{1}{r_{0}}\right) \\
& =\frac{t}{r} \frac{1}{2 y F(y)} r^{\prime}-\frac{t}{r_{0}}\left(\frac{\rho_{0}}{\bar{\rho}_{0}}+\frac{1}{2 y F(y)}-1\right)
\end{aligned}
$$

which gives

$r^{\prime}=\frac{r}{r_{0}}\left\{1+\left(\frac{\rho_{0}}{\bar{\rho}_{0}}-1\right) 2 \sqrt{\ln \left(\frac{r}{r_{0}}\right)} F\left[\sqrt{\ln \left(\frac{r}{r_{0}}\right)}\right]\right\}$ 


\section{The spherically-symmetric derivatives}

First, let $a=\frac{q Q_{\text {tot }}}{\pi m \epsilon_{0}}$. We begin by rewriting $t$ from Eq. $(\mathrm{C} 10)$ as

$$
t=\sqrt{\frac{2 r_{0}^{3}}{a P_{0}}}\left(\tanh ^{-1} y+\frac{r}{r_{0}} y\right)
$$

where $y=\sqrt{1-\frac{r_{0}}{r}}$. So

$$
y^{\prime}=-\frac{1}{2 y r}\left(1-\frac{r_{0}}{r} r^{\prime}\right)
$$

Hence

$$
\left(\tanh ^{-1} y\right)^{\prime}=-\frac{1}{2 y r_{0}}\left(1-\frac{r_{0}}{r} r^{\prime}\right)
$$

and

$$
\left(\frac{r}{r_{0}} y\right)^{\prime}=\frac{1}{2 y r_{0}}\left[2\left(r^{\prime}-\frac{r}{r_{0}}\right)\left(1-\frac{r_{0}}{r}\right)-1+\frac{r_{0}}{r} r^{\prime}\right]
$$

Therefore

$$
\left(\tanh ^{-1} y+\frac{r}{r_{0}} y\right)^{\prime}=\frac{1}{y r_{0}}\left(r^{\prime}-\frac{r}{r_{0}}\right)
$$

Also, similar to Eq. (D4),

$$
\begin{aligned}
\left(\frac{1}{\sqrt{P_{0}}}\right)^{\prime} & =-\frac{1}{2 \sqrt{P_{0}}} \frac{P_{0}^{\prime}}{P_{0}} \\
& =-\frac{3}{2} \frac{1}{\sqrt{P_{0}}} \frac{1}{r_{0}} \frac{\rho_{0}}{\bar{\rho}_{0}}
\end{aligned}
$$

Putting this together we have

$$
0=t^{\prime}=\frac{3}{2} \frac{t}{r_{0}}-\frac{3}{2} \frac{t}{r_{0}} \frac{\rho_{0}}{\bar{\rho}_{0}}+\sqrt{\frac{2 r_{0}^{3}}{a P_{0}}} \frac{1}{y r_{0}}\left(r^{\prime}-\frac{r}{r_{0}}\right)
$$

Solving for $r^{\prime}$ we get

$$
\begin{aligned}
r^{\prime}= & \frac{r}{r_{0}}\left\{1+\frac{3}{2}\left(\frac{\rho_{0}}{\bar{\rho}_{0}}-1\right)\left[\frac{r_{0}}{r} \sqrt{1-\frac{r_{0}}{r}} \tanh ^{-1}\left(\sqrt{1-\frac{r_{0}}{r}}\right)\right.\right. \\
& \left.\left.+1-\frac{r_{0}}{r}\right]\right\}
\end{aligned}
$$

[1] J. D. V. Arbañil, J. P. S. Lemos, and V. T. Zanchin, Incompressible relativistic spheres: Electrically charged stars, compactness bounds, and quasiblack hole configurations, Phys. Rev. D 89, 104054 (2014).
[2] S. Maurya, Y. Gupta, S. Ray, and S. R. Chowdhury, Spherically symmetric charged compact stars, Eur. Phys. J. C 75, 389 (2015).

[3] R. Yousefi, A. B. Davis, J. Carmona-Reyes, L. S. Matthews, and T. W. Hyde, Measurement of net electric charge and dipole moment of dust aggregates in a complex plasma, Phys. Rev. E 90, 033101 (2014).

[4] A. Bacci and A. Rossi, Ultra-short electron bunches by Velocity Bunching as required for plasma wave accelerations, Nucl. Instrum. Methods Phys. Res., Sect. A 740, 42 (2014).

[5] O. Boine-Frankenheim, I. Hofmann, J. Struckmeier, and S. Appel, Artificial collisions, entropy and emittance growth in computer simulations of intense beams, Nucl. Instrum. Methods Phys. Res., Sect. A 770, 164 (2015).

[6] B. Whelan, S. Gierman, L. Holloway, J. Schmerge, P. Keall, and R. Fahrig, A novel electron accelerator for MRILinac radiotherapy, Med. Phys. 43, 1285 (2016).

[7] S. Bernal, B. Beaudoin, I. Haber, T. Koeth, Y. Mo, E. Montgomery, K. Rezaei, K. Ruisard, D. Sutter, H. Zhang et al., Nonlinear dynamics with space-charge in a small electron recirculator, AIP Conf. Proc. 1777, 100003 (2016).

[8] S. Bulanov, T.Z. Esirkepov, F. Kamenets, Y. Kato, A. Kuznetsov, K. Nishihara, F. Pegoraro, T. Tajima, and V. Khoroshkov, Generation of high-quality charged particle beams during the acceleration of ions by high-power laser radiation, Plasma Phys. Rep. 28, 975 (2002).

[9] Y. Fukuda, A. Y. Faenov, M. Tampo, T. Pikuz, T. Nakamura, M. Kando, Y. Hayashi, A. Yogo, H. Sakaki, T. Kameshima et al., Energy Increase in Multi-MeV Ion Acceleration in the Interaction of a Short Pulse Laser with a Cluster-Gas Target, Phys. Rev. Lett. 103, 165002 (2009).

[10] T. Esirkepov, M. Borghesi, S. V. Bulanov, G. Mourou, and T. Tajima, Highly Efficient Relativistic-Ion Generation in the Laser-Piston Regime, Phys. Rev. Lett. 92, 175003 (2004).

[11] A. Kaplan, Coulomb cluster explosion boosted by a quasidc pulse-diagnostic tool and ultimate test of laser fusion efficiency in clusters, arXiv:1503.06368.

[12] P. B. Parks, T. E. Cowan, R. B. Stephens, and E. M. Campbell, Model of neutron-production rates from femtosecond-lasercluster interactions, Phys. Rev. A 63, 063203 (2001).

[13] V. Y. Bychenkov, A. V. Brantov, E. A. Govras, and V. F. Kovalev, Laser acceleration of ions: recent results and prospects for applications, Phys. Usp. 58, 71 (2015).

[14] D. Murphy, R. Speirs, D. Sheludko, C. Putkunz, A. McCulloch, B. Sparkes, and R. Scholten, Detailed observation of space-charge dynamics using ultracold ion bunches, Nat. Commun. 5, 4489 (2014).

[15] A. Gahlmann, S. T. Park, and A. H. Zewail, Ultrashort electron pulses for diffraction, crystallography and microscopy: theoretical and experimental resolutions, Phys. Chem. Chem. Phys. 10, 2894 (2008).

[16] J. H. Booske, R. J. Dobbs, C. D. Joye, C. L. Kory, G. R. Neil, G.-S. Park, J. Park, and R. J. Temkin, Vacuum electronic high power terahertz sources, IEEE Trans. Terahertz Sci. Technol. 1, 54 (2011).

[17] Y. Liu, P. Zhang, S. Chen, and L. Ang, Maximal charge injection of a uniform separated electron pulse train in a 
drift space, Phys. Rev. ST Accel. Beams 18, 123402 (2015).

[18] P. Zhang and Y. Lau, Ultrafast and nanoscale diodes, J. Plasma Phys. 82, 595820505 (2016).

[19] G. H. Jansen, Coulomb Interactions in Particle Beams (Technische Universiteit Delft, Delft, 1988).

[20] M. Reiser, Theory and Design of Charged Particle Beams (John Wiley \& Sons, New York, 1994).

[21] Y. K. Batygin, Self-consistent analysis of three-dimensional uniformly charged ellipsoid with zero emittance, Phys. Plasmas 8, 3103 (2001).

[22] V. Y. Bychenkov and V. Kovalev, Coulomb explosion in a cluster plasma, Plasma Phys. Rep. 31, 178 (2005).

[23] M. Grech, R. Nuter, A. Mikaberidze, P. Di Cintio, L. Gremillet, E. Lefebvre, U. Saalmann, J. M. Rost, and S. Skupin, Coulomb explosion of uniformly charged spheroids, Phys. Rev. E 84, 056404 (2011).

[24] A. E. Kaplan, B. Y. Dubetsky, and P. L. Shkolnikov, Shock Shells in Coulomb Explosions of Nanoclusters, Phys. Rev. Lett. 91, 143401 (2003).

[25] V. Kovalev and V. Y. Bychenkov, Kinetic description of the Coulomb explosion of a spherically symmetric cluster, J. Exp. Theor. Phys. 101, 212 (2005).

[26] I. Last, I. Schek, and J. Jortner, Energetics and dynamics of Coulomb explosion of highly charged clusters, J. Chem. Phys. 107, 6685 (1997).

[27] M. Eloy, R. Azambuja, J. Mendonca, and R. Bingham, Interaction of ultrashort high-intensity laser pulses with atomic clusters , Phys. Plasmas 8, 1084 (2001).

[28] V. P. Krainov and A. S. Roshchupkin, Dynamics of the Coulomb explosion of large hydrogen iodide clusters irradiated by superintense ultrashort laser pulses, Phys. Rev. A 64, 063204 (2001).

[29] J. P. Morrison and E. R. Grant, Dynamics of colliding ultracold plasmas, Phys. Rev. A 91, 023423 (2015).

[30] E. Boella, B. P. Paradisi, A. DAngola, L. O. Silva, and G. Coppa, Study on Coulomb explosions of ion mixtures, J. Plasma Phys. 82, 905820110 (2016).

[31] V. P. Degtyareva, M. A. Monastyrsky, M. Y. Schelev, and V. A. Tarasov, Computer studies on dynamics of ultrashort electron packets in streak tubes and diffractometers, Opt. Eng. 37, 2227 (1998).

[32] B. J. Siwick, J. R. Dwyer, R. E. Jordan, and R. J. Dwayne Miller, Ultrafast electron optics: Propagation dynamics of femtosecond electron packets, J. Appl. Phys. 92, 1643 (2002).

[33] B.-L. Qian and H. E. Elsayed-Ali, Electron pulse broadening due to space charge effects in a photoelectron gun for electron diffraction and streak camera systems, J. Appl. Phys. 91, 462 (2002).

[34] B. W. Reed, Femtosecond electron pulse propagation for ultrafast electron diffraction, J. Appl. Phys. 100, 034916 (2006).

[35] S. Collin, M. Merano, M. Gatri, S. Sonderegger, P. Renucci, J.-D. Ganiere, and B. Deveaud, Transverse and longitudinal space-charge-induced broadenings of ultrafast electron packets, J. Appl. Phys. 98, 094910 (2005).

[36] Z. Tao, H. Zhang, P. Duxbury, M. Berz, and C.-Y. Ruan, Space charge effects in ultrafast electron diffraction and imaging, J. Appl. Phys. 111, 044316 (2012).
[37] J. Portman, H. Zhang, Z. Tao, K. Makino, M. Berz, P. Duxbury, and C.-Y. Ruan, Computational and experimental characterization of high-brightness beams for femtosecond electron imaging and spectroscopy, Appl. Phys. Lett. 103, 253115 (2013).

[38] J. Portman, H. Zhang, K. Makino, C. Ruan, M. Berz, and P. Duxbury, Untangling the contributions of image charge and laser profile for optimal photoemission of highbrightness electron beams, J. Appl. Phys. 116, 174302 (2014).

[39] A. Michalik and J. Sipe, Analytic model of electron pulse propagation in ultrafast electron diffraction experiments , J. Appl. Phys. 99, 054908 (2006).

[40] W. E. King, G. H. Campbell, A. Frank, B. Reed, J. F. Schmerge, B. J. Siwick, B. C. Stuart, and P. M. Weber, Ultrafast electron microscopy in materials science, biology, and chemistry, J. Appl. Phys. 97, 111101 (2005).

[41] E. Hall, S. Stemmer, H. Zheng, Y. Zhu, and G. Maracas, Future of electron scattering and diffraction, US Department of Energy, Washington, DC, Technical Report, 2014.

[42] J. Williams, F. Zhou, T. Sun, K. Chang, K. Makino, M. Berz, P. Duxbury, and C.-Y. Ruan, Active control of bright electron beams with RF optics for femtosecond microscopy, Structural Dynamics 4, 044035 (2017).

[43] A. Valfells, D. Feldman, M. Virgo, P. O'shea, and Y. Lau, Effects of pulse-length and emitter area on virtual cathode formation in electron guns, Phys. Plasmas 9, 2377 (2002).

[44] O. J. Luiten, S. B. vanderGeer, M. J. deLoos, F. B. Kiewiet, and M. J. vanderWiel, How to Realize Uniform ThreeDimensional Ellipsoidal Electron Bunches, Phys. Rev. Lett. 93, 094802 (2004).

[45] R. D. Miller, Femtosecond crystallography with ultrabright electrons and X-rays: Capturing chemistry in action, Science 343, 1108 (2014).

[46] R. Srinivasan, V. Lobastov, C.-Y. Ruan, and A. Zewail, A new development for the $4 \mathrm{~d}$ determination of transient molecular structures, Helv. Chim. Acta 86, 1761 (2003).

[47] C.-Y. Ruan, Y. Murooka, R. K. Raman, R. A. Murdick, R. J. Worhatch, and A. Pell, The development and applications of ultrafast electron nanocrystallography, Microsc. Microanal. 15, 323 (2009).

[48] T. vanOudheusden, P. L. E. M. Pasmans, S. B. vanderGeer, M. J. deLoos, M. J. vanderWiel, and O. J. Luiten, Compression of Subrelativistic Space-Charge-Dominated Electron Bunches for Single-Shot Femtosecond Electron Diffraction, Phys. Rev. Lett. 105, 264801 (2010).

[49] G. Sciaini and R. D. Miller, Femtosecond electron diffraction: heralding the era of atomically resolved dynamics, Rep. Prog. Phys. 74, 096101 (2011).

[50] P. Musumeci, J. Moody, C. Scoby, M. Gutierrez, M. Westfall, and R. Li, Capturing ultrafast structural evolutions with a single pulse of $\mathrm{MeV}$ electrons: Radio frequency streak camera based electron diffraction, J. Appl. Phys. 108, 114513 (2010).

[51] S. Weathersby et al., Mega-electron-volt ultrafast electron diffraction at SLAC National Accelerator Laboratory, Rev. Sci. Instrum. 86, 073702 (2015).

[52] Y. Murooka, N. Naruse, S. Sakakihara, M. Ishimaru, J. Yang, and K. Tanimura, Transmission-electron diffraction 
by $\mathrm{MeV}$ electron pulses, Appl. Phys. Lett. 98, 251903 (2011).

[53] J. Rosenzweig, A. Cook, R. England, M. Dunning, S. Anderson, and M. Ferrario, Emittance compensation with dynamically optimized photoelectron beam profiles, Nucl. Instrum. Methods Phys. Res., Sect. A 557, 87 (2006).

[54] P. Musumeci, J. T. Moody, R. J. England, J. B. Rosenzweig, and T. Tran, Experimental Generation and Characterization of Uniformly Filled Ellipsoidal Electron-Beam Distributions, Phys. Rev. Lett. 100, 244801 (2008).

[55] J. Williams, F. Zhou, T. Sun, P. Duxbury, S. Lund, B. Zerbe, and C.-Y. Ruan, Phase-space characterization and optimization of high-brightness electron beams for femtosecond imaging and spectroscopy near the single-shot limit, Bull. Am. Phys. Soc. 62 (2017).

[56] M. Berz, H. Hoffmann, and H. Wollnik, COSY 5.0-The fifth order code for corpuscular optical systems, Nucl. Instrum. Methods Phys. Res., Sect. A 258, 402 (1987).

[57] H. Zhang, J. Portman, Z. Tao, P. Duxbury, C.-Y. Ruan, K. Makino, and M. Berz, The differential algebra based multiple level fast multipole algorithm for $3 \mathrm{~d}$ space charge field calculation and photoemission simulation, Microsc. Microanal. 21, 224 (2015).

[58] A. Friedman, R. H. Cohen, D. P. Grote, S. M. Lund, W. M. Sharp, J.-L. Vay, I. Haber, and R. A. Kishek, Computational Methods in the Warp Code Framework for Kinetic Simulations of Particle Beams and Plasmas, IEEE Trans. Plasma Sci. 42, 1321 (2014).

[59] V. R. Morrison, R. P. Chatelain, C. Godbout, and B. J. Siwick, Direct optical measurements of the evolving spatio-temporal charge density in ultrashort electron pulses , Opt. Express 21, 21 (2013).

[60] Y. Li and J. W. Lewellen, Generating a Quasiellipsoidal Electron Beam by 3D Laser-Pulse Shaping, Phys. Rev. Lett. 100, 074801 (2008).

[61] E. Lessner, X. Wang, and P. Musumeci, SLAC National Accelerator Laboratory Report of the Basic Energy Sciences Workshop on the Future of Electron Sources, 2016.

[62] K. Boyer, T. S. Luk, J. C. Solem, and C. K. Rhodes, Kinetic energy distributions of ionic fragments produced by subpicosecond multiphoton ionization of $\mathrm{N}_{2}$, Phys. Rev. A 39, 1186 (1989).

[63] T. Wangler, K. Crandall, R. Mills, and M. Reiser, Relation between Field Energy and RMS Emittance in Intense Particle Beams, IEEE Trans. Nucl. Sci. 32, 2196 (1985).

[64] K. Yee, Numerical solution of initial boundary value problems involving maxwell's equations in isotropic media, IEEE Trans. Antennas Propag. 14, 302 (1966).

[65] V. Y. Bychenkov and V. F. Kovalev, Relativistic coulomb explosion of spherical microplasma, JETP Lett. 94, 97 (2011).

[66] J. W. Luginsland, Y. Y. Lau, and R. M. Gilgenbach, TwoDimensional Child-Langmuir Law, Phys. Rev. Lett. 77, 4668 (1996).

[67] R. L. Gluckstern, Analytic Model for Halo Formation in High Current Ion Linacs, Phys. Rev. Lett. 73, 1247 (1994).

[68] T. Wangler, K. Crandall, R. Ryne, and T. Wang, Particlecore model for transverse dynamics of beam halo, Phys. Rev. ST Accel. Beams 1, 084201 (1998).

[69] W. R. Inc., Mathematica, Version 11.1, Champaign, IL, 2017. 